Check for updates

Cite this: J. Mater. Chem. C, 2020, 8, 17237

Received 28th August 2020, Accepted 17th November 2020

DOI: $10.1039 / \mathrm{dOtc0} 4110 f$

rsc.li/materials-c

\section{Ring fusion in tetrathienylethene cored perylene diimide tetramers affords acceptors with strong and broad absorption in the near-UV to visible region $\dagger$}

\author{
Qiao He, (D) $\ddagger^{\mathrm{a}}$ Flurin D. Eisner, (D) $\ddagger^{\mathrm{b}}$ Drew Pearce, ${ }^{\mathrm{b}}$ Thomas Hodsden, ${ }^{\mathrm{a}}$ \\ Elham Rezasoltani, ${ }^{\mathrm{b}}$ Daniel Medranda, ${ }^{\mathrm{b}}$ Zhuping Fei, ${ }^{\mathrm{c}}$ Jenny Nelson (D) *b and \\ Martin Heeney (iD) *a
}

\begin{abstract}
In this work, we designed and synthesized two novel perylene diimide (PDI) tetramers based on a tetrathienylethene core, named TTE-PDI4 and FTTE-PDI4, and investigated their application as nonfullerene acceptors for organic photovoltaics. The free rotation of PDIs and adjacent thiophene units renders TTE-PDI4 with a highly twisted molecular geometry. The ring fusion of TTE-PDI4 yields FTTE-PDI4, a more rigid molecule with increased intramolecular stacking. Interestingly, TTE-PDI4 and FTTE-PDI4 possess similar energy levels but very different UV-Vis absorptions, with the latter showing strong broad-band absorption with multiple sharp peaks in the 300-600 nm region. Through timedependent density functional theory (TD-DFT) calculations, we show that this broad absorption spectrum in FTTE-PDI4 arises from the combination of multiple bright transitions in the visible region with a strong vibronic progression, tentatively assigned to the dominant $\mathrm{C}=\mathrm{C}$ stretching mode. TTE-PDI4, despite having a lower energy absorption onset, shows weaker absorption at long wavelengths. Due to its higher absorption as well as its increased rigidity, FTTE-PDI4 shows a higher photocurrent and hence a higher power conversion efficiency (PCE), of $6.6 \%$, when blended with the polymer donor PFBDB-T than TTE-PDI4 based blends (PCE of 3.8\%). The greater rigidity of FTTE-PDI4 is likely to contribute to the good fill factor of the blend devices. Potential for further improvement through reducing voltage losses is identified.
\end{abstract}

\section{Introduction}

Among various photovoltaic energy-conversion technologies, organic photovoltaics (OPVs) have become one of the fastestgrowing areas over the past few years, attributed to the successful design and development of non-fullerene acceptors (NFAs). ${ }^{1-6}$ Such materials have helped to overcome some of the limitations of fullerene based acceptors $\left(\mathrm{PC}_{61} \mathrm{BM}\right.$ and $\mathrm{PC}_{71} \mathrm{BM}$ ) including weak absorption in the visible region, elevated synthetic difficulty in tuning the electronic/optical properties, and poor morphology stability. ${ }^{7-9}$ These issues have severely retarded the further development of fullerene based

\footnotetext{
${ }^{a}$ Department of Chemistry and Centre for Processable Electronics, Imperial College London, London W12 OBZ, UK. E-mail: m.heeney@imperial.ac.uk

${ }^{b}$ Department of Physics and Centre for Processable Electronics, Imperial College London, London SW7 2AZ, UK. E-mail: jenny.nelson@imperial.ac.uk ${ }^{c}$ Institute of Molecular Plus, Tianjin Key Laboratory of Molecular Optoelectronic Science, Tianjin University, Tianjin 300072, P. R. China

$\dagger$ Electronic supplementary information (ESI) available. See DOI: 10.1039/ dotc04110f

\$ These authors contributed equally to this work.
}

OPVs, and thus have accelerated the development of NFAs as an alternative strategy. Compared to their fullerene counterparts, there are more available building blocks to prepare NFAs and this synthetic flexibility affords more readily tunable optoelectronic properties.

With regards to the various types of electron-withdrawing units to construct NFAs, perylene diimide (PDI) has been among the earliest studied and most promising. ${ }^{10-15}$ Pioneering studies on the PDI monomer and its derivatives found that the tendency of the coplanar PDI monomers to strongly aggregate resulted in micrometer-sized phase-separated domains in bulk-heterojunction blends, leading to low OPV device efficiencies. ${ }^{16,17}$ Disrupting molecular planarity by intramolecular twisting has been found to be a useful approach to suppress the formation of large aggregates and improve performance. ${ }^{18-21}$ By connecting two PDI monomers via a spacer or linking more PDIs with a central core, PDI dimers, trimers and tetramers have been prepared with a twisted 3D or quasi-3D geometry, resulting in improved OPV device performance. The spacers can be simply a single or double bond, an aromatic ring or a larger conjugated central core. ${ }^{22-27}$ Although 
the disruption of planarity is generally beneficial, excessive molecular twisting can be detrimental to charge transport and, consequently, high structural non-planarity of PDI-based acceptors can result in reduced device performance..$^{27-29}$ To tackle this issue, ring fusion has been proposed in several studies to finely tune the intermolecular $\pi-\pi$ stacking. ${ }^{30-34}$ Concomitantly, evidence also suggests that ring fusion blue-shifts absorption onset, enhances absorption strength, and up-shifts the lowest unoccupied molecular orbital (LUMO) energy level, which can be beneficial for overall OPV device performance. ${ }^{30,35}$ Through the employment of geometry twisting and aromatic ring fusion, currently the power conversion efficiency (PCE) of the best performing PDI-based NFAs have been enhanced to over $10 \% .^{35,36}$

We were particularly interested by the observations that ring fusion can lead a blue-shifted absorption, since wide band gap NFA absorbers with a strong absorption in the green to near-UV region are of interest for the front cell in tandem devices or for smart window applications. ${ }^{37,38}$ Ring fusion in the bay region of the PDI has typically been achieved by an oxidative ring closure with an electron rich heterocycle like thiophene or selenophene, ${ }^{30,35}$ and in order to prevent excessive aggregation it should be combined with the twisting approach. As such, we identified tetrathienylethene (TTE) as a potentially interesting central core for the attachment of four PDI arms, combining the requisite thienyl groups for ring fusion with a non-planar geometry. TTE has previously been primarily investigated in the field of aggregation-induced emission (AIE). ${ }^{39,40}$ Although the emissive properties are not the focus of this paper, we note that other AIE cores like tetraphenylethene (TPE) have been used for the attachment of PDI arms, although the resulting TPE-PDI4 was not able to undergo ring fusion with the PDI. ${ }^{25}$ Here we report the synthesis of two new non-fused and fused PDI tetramers (TTE-PDI4 and FTTE-PDI4) based on the tetrathienylethene core and investigate their performance in organic photovoltaic devices. For the non-fused TTE-PDI4, freely rotating single bonds between thiophene and the central ethene, as well as thiophene and the PDI, induces strong steric hindrance leading to a highly twisted molecular structure. In comparison, DFT calculations suggest the fused tetramer, FTTE-PDI4, displays a more ordered geometry with the interlocking of thiophenes and PDI units. The ring fusion leads to an up-shifted LUMO level, and an intense and extremely broad absorption spectrum at visible to near-UV wavelengths. These are found by time-dependent DFT to arise from multiple bright electronic transitions. These beneficial changes contribute to higher short-circuit current density $\left(J_{\mathrm{SC}}\right)$ and improved power conversion efficiency from $3.8 \%$ (TTE-PDI4) to $6.6 \%$ (FTTE-PDI4) in solar cell devices, showing that careful design of the central core on PDI tetramers can lead to promising wide band gap acceptors.

\section{Results and discussion}

\section{Synthesis of TTE-PDI4 and FTTE-PDI4}

The synthetic route to TTE-PDI4 and FTTE-PDI4 is presented in Scheme 1. The intermediates DTK and TTE were synthesized according to a previous report. ${ }^{41}$ The TTE-Bpin 4 was prepared by tetraborylation of TTE via an Ir-catalyzed reaction. Subsequent fourfold Suzuki coupling reaction between TTE-Bpin4 and C6-PDI-Br afforded the first product TTE-PDI4 in 40\% yield. By subsequent treatment with $\mathrm{FeCl}_{3} / \mathrm{CH}_{3} \mathrm{NO}_{2}$, an oxidative cyclization reaction occurred between the PDI subunits and
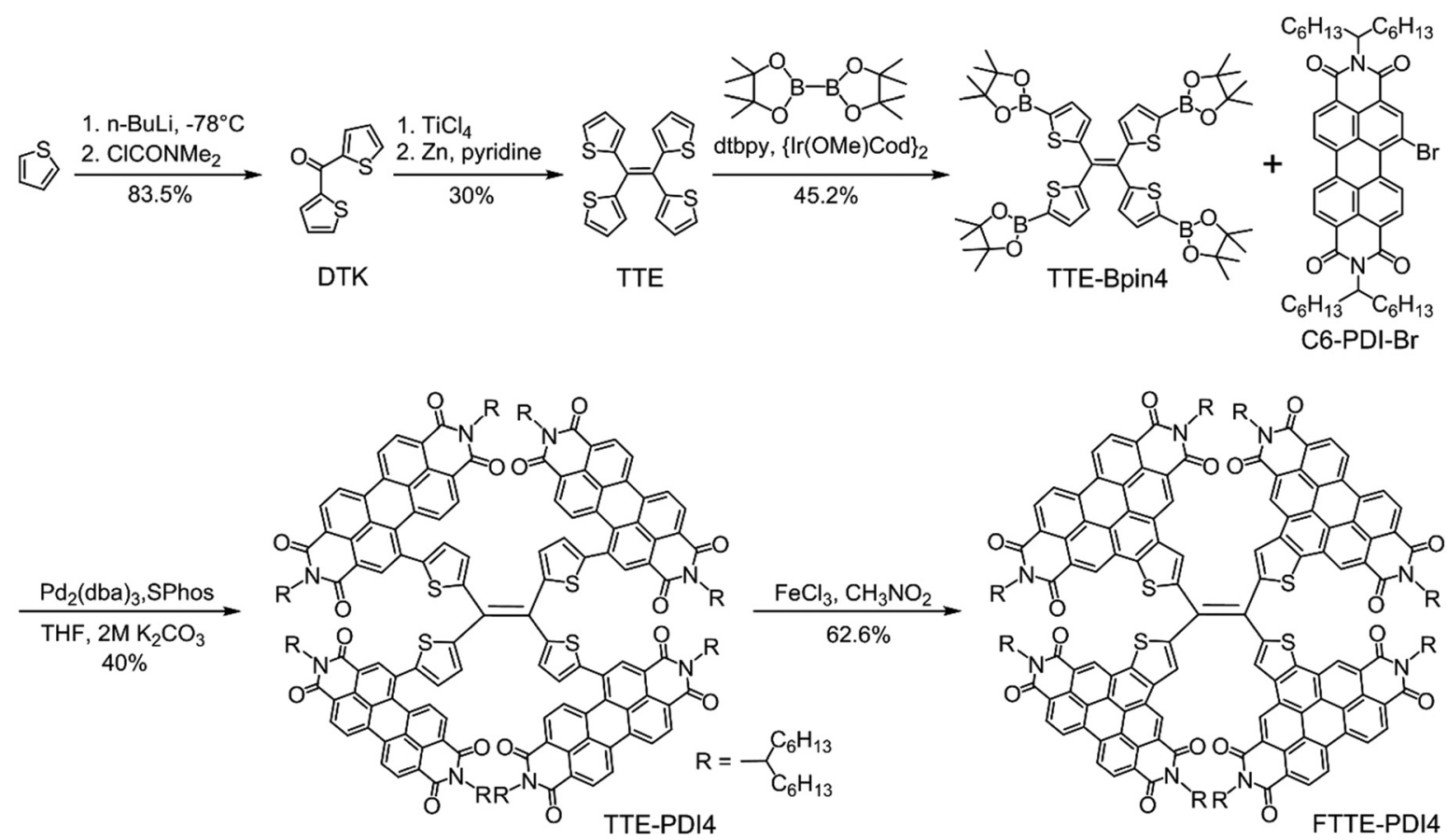

Scheme 1 Synthetic route to TTE-PDI4 and FTTE-PDI4. 
their neighboring thiophene spacers, affording the fused product FTTE-PDI4 in $62.6 \%$ yield.

TTE-PDI4 and FTTE-PDI4 were purified by a combination of column chromatography followed by preparative recycling gelpermeation chromatography (GPC) to remove non-tetrameric by-products (see ESI $\dagger$ ). The identities of TTE-PDI4 and FTTEPDI4 were confirmed by MALDI-TOF mass spectrometry (Fig. S25 and S27, ESI $\dagger$ ). Room temperature ${ }^{1} \mathrm{H}$ NMR spectra were broad and poorly resolved, similar to other PDI tetramers, ${ }^{15,34,35}$ as a result of the restricted rotation of the PDI subunits and presence of multiple conformers. Heating FTTE-PDI4 solutions at $40{ }^{\circ} \mathrm{C}, 80{ }^{\circ} \mathrm{C}$ and $120{ }^{\circ} \mathrm{C}$ resulted in improved resolution (Fig. S24, ESI $\dagger$ ), particularly of the protons on the outer shell of the molecule which were clearly resolved as sets of doublets. The inner protons nearer the TTE core were less well resolved, possibly due to more restricted movement.

As such, it is difficult to completely rule out the presence of some incompletely fused material, although the significant change in optical properties (vide infra) suggests that this would only be a minor impurity if present.

Both compounds are readily soluble in common organic solvents such as chloroform and chlorobenzene at room temperature. The structures of the two PDI tetramers were characterized and confirmed by MALDI-TOF MS, ${ }^{1} \mathrm{H}$ NMR, and ${ }^{13} \mathrm{C}$ NMR. Both TTE-PDI4 and FTTE-PDI4 exhibited good thermal stability, with thermal decomposition temperatures $\left(T_{\mathrm{d}}\right)$ of $320{ }^{\circ} \mathrm{C}$ and $375{ }^{\circ} \mathrm{C}$, respectively, measured by thermogravimetric analysis (TGA, at 5\% weight loss, Fig. S1, ESI $\dagger$ ). In addition, in the range of $20{ }^{\circ} \mathrm{C}$ to $280{ }^{\circ} \mathrm{C}$, no obvious crystallization or melting transitions could be observed for both PDI tetramers, indicating their weak crystallization tendency (Fig. S2, ESI $\dagger$ ).

\section{DFT and TD-DFT calculations}

To investigate the molecular structural geometries and frontier molecular orbitals of TTE-PDI4 and FTTE-PDI4, DFT calculations
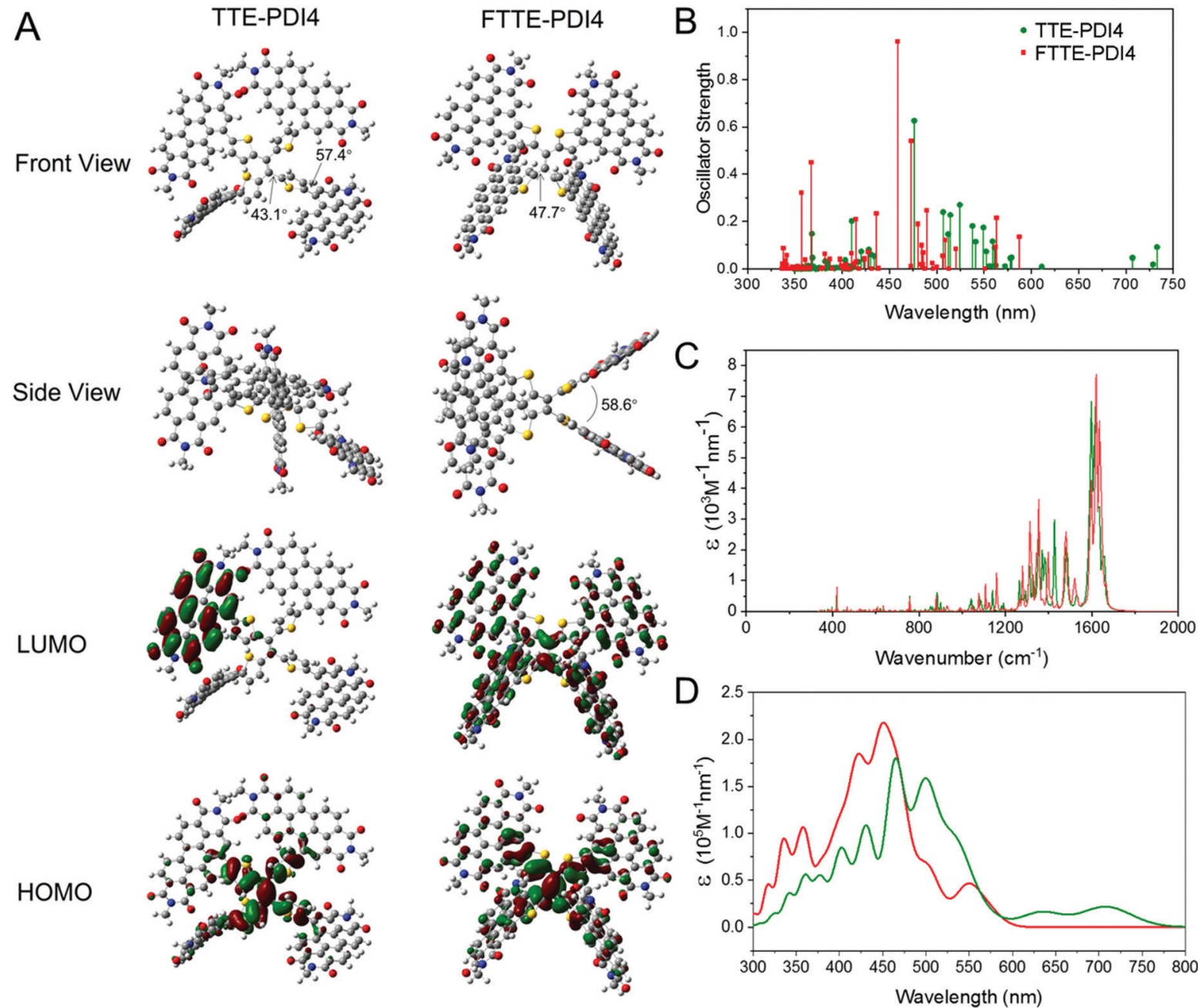

Fig. 1 (A) Optimized geometry (front and side views) and frontier molecular orbitals of TTE-PDI4 and FTTE-PDI4 using DFT calculation at the B3LYP/6$31 \mathrm{G}$ level. (B) Oscillator strength $(\varepsilon)$ calculations of the excited state transitions to the ground state and (C) extinction coefficients $(\varepsilon)$ from vibrational transitions calculated using TD-DFT, with the B3LYP functional and 6-311g basis set; 100 states were calculated and broadened with a half-width at half height of $0.2 \mathrm{eV}$. Frequency calculations were also performed to obtain the vibrational transitions. (D) Extinction coefficient calculations based on a Franck-Condon lineshape applied to the electronic transitions. 
were performed using the B3LYP functional and 6-31G basis set, with all alkyl chains replaced by methyl groups for computational simplicity. Both TTE-PDI4 and FTTE-PDI4 are predicted to exhibit highly twisted molecular geometries, with the former exhibiting a propeller type arrangement in which each arm is twisted with respect to the central ethene, with dihedral angles between the ethene and thiophene of $43^{\circ}$, and $57^{\circ}$ between the thiophene and PDIs. As expected the ring fusion in FTTE-PDI4 eliminates the rotational freedom between the thiophenes and PDI units (Fig. 1A and Fig. S3, ESI $\dagger$ ), and the predicted structure becomes more saddle shaped, with two PDI-Th planes at each end of the central ethene. There remains a large twist between the PDI-Th and the central ethene of $48^{\circ}$, with the two PDI-Th planes rigidly held in space, with the plane of one rotated by an angle of $59^{\circ}$ with respect to the other. With regards to the frontier molecular orbitals, though the HOMOs are localised on the TTE central core for both fused and non-fused NFAs, the delocalization of LUMOs shows significant difference. In TTE-PDI4, the near degenerate LUMO and LUMO+1 orbitals localize on different PDI units. In contrast, the LUMO of FTTE-PDI4 delocalizes through the entire molecular backbone as the fused molecule becomes more planar and rigid. The internal reorganization energies upon positive polaron formation were calculated to be $0.105 \mathrm{eV}$ and $0.116 \mathrm{eV}$ for FTTE-PDI4 and TTE-PDI4, respectively. The lower reorganization energy for the FTTE-PDI4 is consistent with its increased rigidity.

In order to investigate the effect of the different structural geometries of the two molecules on their absorption profile, we additionally carried out time-dependent DFT (TD-DFT) calculations. Fig. $1 \mathrm{~B}$ and $\mathrm{C}$ thus show the electronic and vibrational structure calculations, respectively. The results firstly show that we expect TTE-PDI4 to have a smaller band gap than FTTE-PDI4 by around $0.1 \mathrm{eV}$ due to a weak low energy transition that appears in the extinction spectrum as a long and shallow tail. Secondly, FTTE-PDI4 shows strong absorption at low wavelengths due to several bright electronic transitions in the visible, in agreement with previous studies, ${ }^{42}$ while lacking the weak low energy transition that creates the absorption tail in TTE-PDI4. Thirdly, vibrational structure calculations show that there is a strong vibrational band at $1600 \mathrm{~cm}^{-1}$ that is present in both molecules. The mode is assigned to the $\mathrm{C}=\mathrm{C}$ bond stretch within the PDI units, as shown in Fig. S5 (ESI $\dagger$ ). When a mode of this frequency is used to model the vibronic progression of the extinction spectra of the two molecules (Fig. 1D) a spectrum with multiple peaks across the visible results. We note that broad absorption features at low wavelengths have often been reported in the absorption spectra of PDI-based molecules, in particular in fused PDIs $;^{35,43}$ our calculations suggest that these features may result from the combination of multiple close lying electronic transitions with a strong vibronic progression.

\section{Optical and electronic properties}

The measured optical and electronic properties of TTE-PDI4 and FTTE-PDI4 are summarized in Table 1. As presented in Fig. 2A and B, the UV-Vis absorption spectra of TTE-PDI4 and FTTE-PDI4 films also exhibit significant differences. Both the solution and film absorptions of TTE-PDI4 exhibit an absorption peak in the region of 400-650 nm, similar to the PDI monomer, while those of FTTE-PDI4 show multiple distinctive sharp peaks in the region of 350-600 $\mathrm{nm}$, and are in good agreement with the calculated absorption spectra (Fig. 1D). This comparison is also in agreement with the increased number of bright electronic transitions in the visible region in FTTE-PDI4 compared with TTE-PDI4, as identified from TD-DFT calculations. The relative intensities of the $0-1$ and 0-0 transitions $\left(I_{0-1} / I_{0-0}\right)$ have been previously identified as indicative of the $\mathrm{H}$-aggregation of PDI chromophores. ${ }^{4-45}$ Since aggregation is inhibited by the non-planar structure of the molecules, the high $I_{0-1}(450 \mathrm{~nm}) / I_{0-0}(500 \mathrm{~nm})=1.15$ of FTTE-PDI4 in films thus suggests a degree of long-range coupling of the PDI chromophores possibly related to the rigid arrangement of the PDI units leading to H-aggregation of the PDI chromophores. ${ }^{46}$ Notably, fused FTTE-PDI4 also shows higher absorptivity in the 300-450 region $\mathrm{nm}$ and a blueshifted absorption onset compared to TTE-PDI4, resulting in a higher band gap of 2.04 compared to $1.90 \mathrm{eV}$ (calculated by the crossing point between absorption and emission, Fig. S6, $\mathrm{ESI} \dagger$ ), in agreement with the theoretical calculations.

In addition, the maximum extinction coefficients of FTTE-PDI4 in solution and in film were calculated to be $1.41 \times 10^{5} \mathrm{M}^{-1} \mathrm{~cm}^{-1}$ and $3.77 \times 10^{4} \mathrm{~cm}^{-1}$, respectively, higher than those of TTE-PDI4 $\left(1.19 \times 10^{5} \mathrm{M}^{-1} \mathrm{~cm}^{-1}\right.$ and $\left.3.55 \times 10^{4} \mathrm{~cm}^{-1}\right)$. The minor differences in the absorption spectra between solution and film further suggest that intermolecular packing is weak for both molecules. This is further confirmed by absence of any change in solution absorption measurements as a function of concentration (Fig. S10, ESI $\dagger$ ). This additionally suggests that the breadth of the absorption of FTTE-PDI4, at over $50 \%$ of maximum intensity from 300 to $600 \mathrm{~nm}$, must be due to the multiple bright electronic states of the molecules identified by TD-DFT rather than due to intermolecular interactions.

The electrochemical properties of these two compounds were investigated by cyclic voltammetry of thin films, with the

Table 1 Optical and electrochemical properties of TTE-PDI4 and FTTE-PDI4

\begin{tabular}{|c|c|c|c|c|c|c|c|c|}
\hline & $\lambda_{\max , \mathrm{sol}}(\mathrm{nm})$ & $\alpha_{\max , \mathrm{sol}}\left(\mathrm{M}^{-1} \mathrm{~cm}^{-1}\right)$ & $\lambda_{\max , \text { film }}(\mathrm{nm})$ & $\alpha_{\max , \text { film }}\left(\mathrm{cm}^{-1}\right)$ & $E_{\mathrm{g}, \mathrm{opt}}{ }^{a}(\mathrm{eV})$ & $E_{\mathrm{g}, \text { electro }}{ }^{b}(\mathrm{eV})$ & $\operatorname{LUMO}^{c}(\mathrm{eV})$ & $\operatorname{HOMO}^{c}(\mathrm{eV})$ \\
\hline TTE-PDI4 & 525 & $1.19 \times 10^{5}$ & 530 & $3.55 \times 10^{4}$ & 1.90 & 2.20 & -3.74 & -5.94 \\
\hline FTTE-PDI4 & 429 & $1.41 \times 10^{5}$ & 424 & $3.77 \times 10^{4}$ & 2.00 & 2.29 & -3.68 & -5.97 \\
\hline
\end{tabular}

${ }^{a}$ Determined by the absorption onset of the films. ${ }^{b}$ Electrochemical band gap, determined by (LUMO-HOMO). ${ }^{c}$ Estimated from the reduction and oxidation onsets of the CV curves. 
A

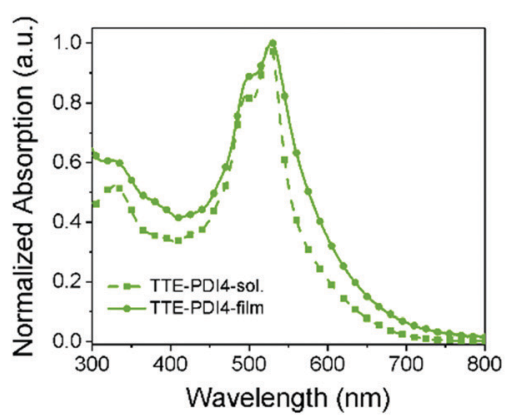

C

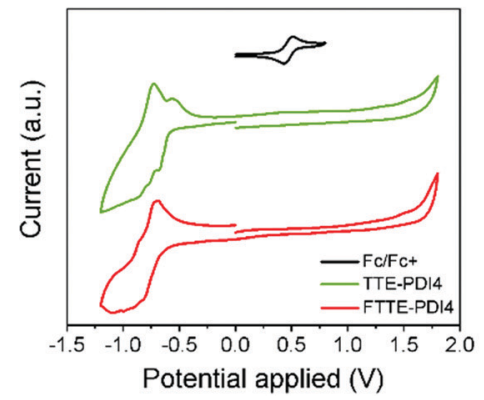

B

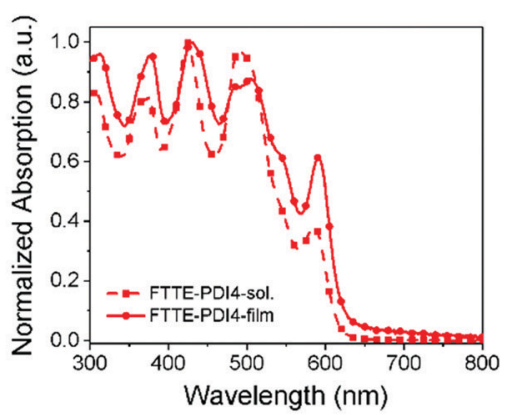

D

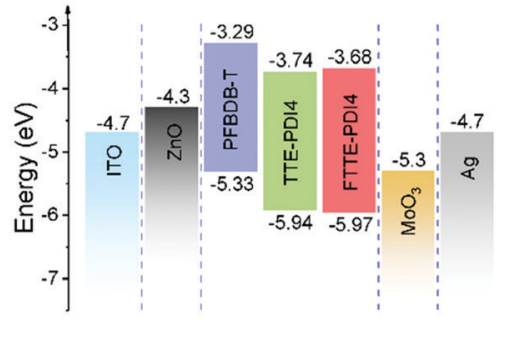

Fig. 2 Normalized absorption spectra of (A) TTE-PDI4 and (B) FTTE-PDI4 in chloroform solution (10 $\left.{ }^{-5} \mathrm{M}\right)$ and as pristine films. (C) Cyclic voltammetry curves of TTE-PDI4 and FTTE-PDI4 films. (D) Energy level diagram of the OPV device.

LUMO/HOMO levels of TTE-PDI4 and FTTE-PDI4 (Fig. 2C) estimated to be similar at $-3.74 /-5.94 \mathrm{eV}$ and $-3.68 / 5.97 \mathrm{eV}$, respectively from the onset of the reduction and oxidation peaks. The slight upshift of the LUMO level upon ring fusion is in agreement with the DFT calculations and with other studies on fused PDIs. ${ }^{30,34,35}$

\section{OPV performance}

To evaluate the photovoltaic performance of these two compounds, OPV devices were fabricated with an inverted structure of ITO/ZnO/polymer donor:TTE-PDI4 or FTTE-PDI4/ $\mathrm{MoO}_{3} / \mathrm{Ag}$ (Fig. 2D). To begin with, several high performance polymers (PTB7-th, ${ }^{47}$ PffBT4T-2OD, ${ }^{48}$ PBDB-T, ${ }^{49}$ PFBDB-T, ${ }^{6}$ and P4FBDB$\mathrm{T}^{50}$ ) were used to fabricated devices with FTTE-PDI4 to determine the most suitable donor.

Reasonably high efficiencies $(\sim 5 \%)$ were achieved with PTB7-Th, PBDB-T and PFBDB-T as the donor (Table S1, ESI $\dagger$ ), with devices with PFBDB-T showing the highest efficiency; we therefore selected this polymer to optimize fabrication conditions with both TTE-PDI4 and FTTE-PDI4 blends. The optimized photovoltaic parameters and typical $J-V$ characteristics of the PFBDB-T:TTE-PDI4 and PFBDB-T:FTTE-PDI4 devices are presented in Fig. 3A and Table 2. The best performance with PFBDB-T:FTTE-PDI4 devices was achieved with a $1: 2$ blend ratio, $2 \%$ 1-chloronaphthalene $(\mathrm{CN})$ as solvent additive and annealing at $140{ }^{\circ} \mathrm{C}$, showing an average PCE of $6.4 \%$ (best

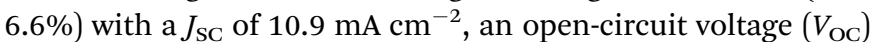
of $1.0 \mathrm{~V}$, and a FF of 0.59 . The relatively large acceptor fraction required for maximum device performance is in agreement with weak intermolecular packing of the molecules (evidenced by the absorption data above) and the low crystallinity (evidenced by featureless DSC spectra). Non-crystalline acceptor molecules generally need to be added in greater excess than crystalline acceptors to achieve percolating networks and phase-pure domains for charge separation. Interestingly, a high PCE was also maintained in as-cast cells without additive and thermal annealing, with such devices showing a lower $J_{\mathrm{SC}}$ but higher $V_{\mathrm{OC}}$ and FF of $1.02 \mathrm{~V}$ and 0.63 , respectively. We note, also, that the highest FF (0.64) is achieved in a $1: 1.5$ blend ratio, which is amongst the highest reported for PDI-based acceptors. The PFBDB-T:TTE-PDI4 based active layers were also systematically optimized under various conditions, with a donor: acceptor ratio of $1: 1.5$, no additive and thermal annealing at $140{ }^{\circ} \mathrm{C}$ leading to the optimized average PCE of the TTE-PDI4 based OPVs of 3.8\%, with inferior current density and FF to the FTTE-PDI4 based OPV devices. A similar $V_{\text {OC }}$ was observed for TTE-PDI4 and FTTE-PDI4 based OPVs, in spite of the slightly higher-lying LUMO levels for the latter. Moreover, external quantum efficiencies (EQEs) of the optimal devices were measured to verify the $J_{\mathrm{SC}}$ values obtained from the $J-V$ measurement (Fig. 3B). The $J_{\mathrm{SC}}$ values integrated from EQE are 7.4 and $11.9 \mathrm{~mA} \mathrm{~cm} \mathrm{~cm}^{-2}$, both in less than $10 \%$ deviation from $J_{\mathrm{SC}}$ measured from the TTE-PDI4 and FTTE-PDI4 based OPV devices. The PFBDB-T:FTTE-PDI4 devices exhibit stronger photo-response in the spectral range between 350 and $600 \mathrm{~nm}$, with a higher maximum EQE of $72 \%$ than the PBDB-T:TTE-PDI4 devices $\left(\mathrm{EQE}_{\max }=56 \%\right)$, in agreement with the stronger absorption of FTTE-PDI4 compared to TTE-PDI4 in that region. This indicates FTTE-PDI4 contributes more to the charge generation and $J_{\mathrm{SC}}$ than TTE-PDI4 as electron acceptor in their corresponding blends.

Using the measured EQE edge (Fig. 3C), we then calculated the photovoltaic band gap energy $\left(E_{\mathrm{g}}^{\mathrm{PV}}\right)$, using the method proposed by Rau, ${ }^{51}$ for both blends to be $1.84 \mathrm{eV}$. These values 

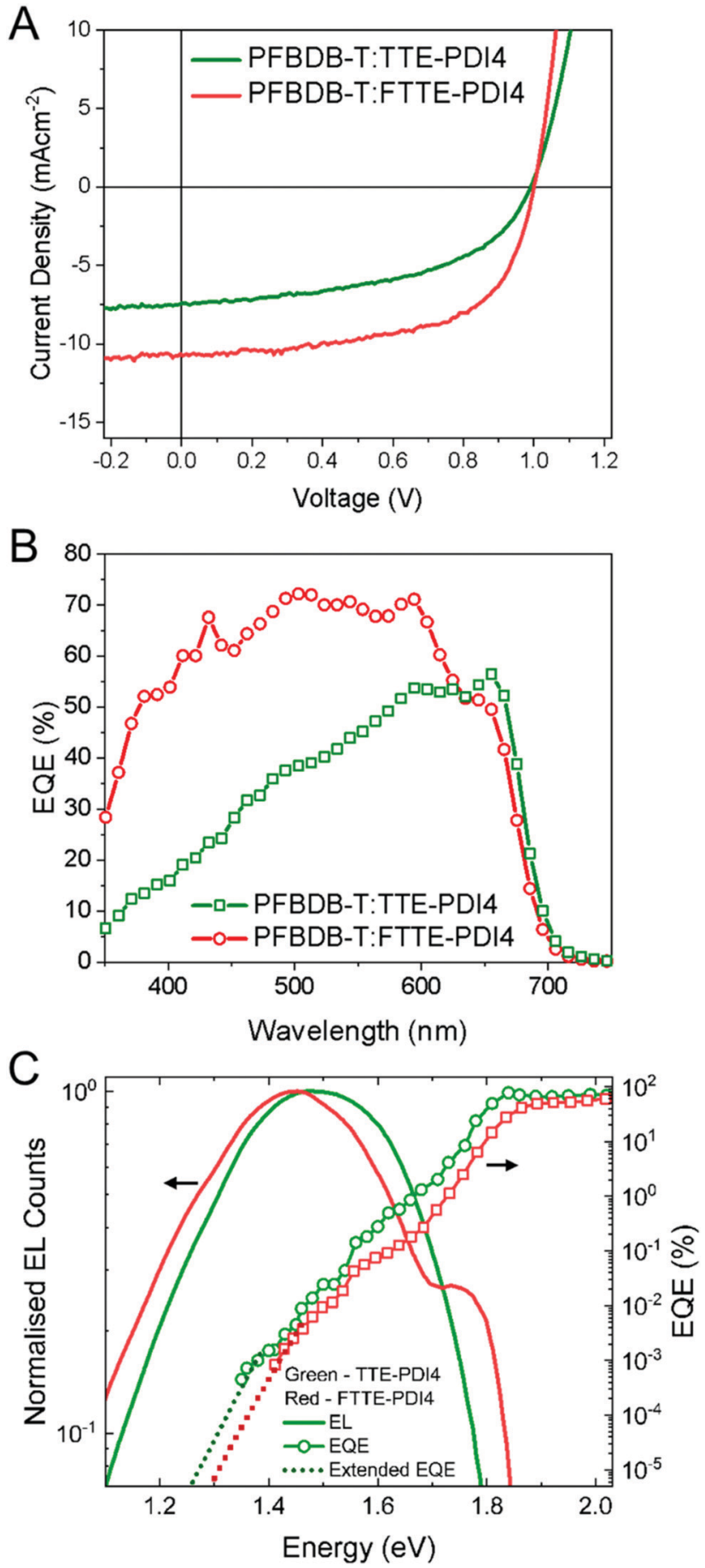

Fig. $3 J-V$ curves (A) and EQEs (B) for the optimized devices based on the PFBDB-T:TTE-PDI4 and PFBDB-T:FTTE-PDI4 blends. (C) Electroluminescence (solid lines), external quantum efficiency (line and symbols), and extended external quantum efficiency (symbols) calculated using reciprocity from the EL spectra of PFBDB-T:TTE-PDI4 (green) and PFBDBT:FTTE-PDI4 (red) blend devices.

suggested high voltage losses $\left(E_{\mathrm{g}}^{\mathrm{PV}} / q-V_{\mathrm{OC}}\right)$ of 0.85 and $0.84 \mathrm{~V}$ for PFBDB-T:TTE-PDI4 and PFBDB-T:FTTE-PDI4 devices, respectively, where $q$ is electronic charge. In order to better understand the origin of these high losses, we therefore performed a voltage-loss analysis of the optimized blends by employing electroluminescence (EL) spectra to extend the measured sub-bandgap EQE spectra (Fig. 3C), using the reciprocity relation between light absorption and light emission, ${ }^{52,53}$ to calculate the open-circuit voltage in the radiative limit $\left(V_{\mathrm{OC}, \mathrm{rad}}\right) . V_{\mathrm{OC} \text {,rad }}$ is the ideal $V_{\mathrm{OC}}$ that a device can achieve when there is only radiative recombination, with the difference between $V_{\text {OC,rad }}$ and the measured $V_{\text {OC }}$ of the device then being defined as the voltage loss due to non-radiative recombination, $\Delta V_{\mathrm{OC}, \mathrm{nrad}}$. The $V_{\mathrm{OC}, \mathrm{rad}}$ and $\Delta V_{\mathrm{OC}, \mathrm{nrad}}$ values for the optimized devices are shown in Table 2 and we also note that the maximum ideal $V_{\mathrm{OC}}$ in the Shockley-Queisser limit $\left(V_{\text {OC,sq }}\right)$ achievable by a device with the measured band gap of $1.84 \mathrm{eV}$ is $1.54 \mathrm{~V}$.

We firstly draw attention to the fact, then, that the difference between $V_{\mathrm{OC} \text {,sq }}$ and $V_{\mathrm{OC} \text {,rad }}$ is large for both blends, which suggests that losses due a broad absorption edge are large; this is expected given the large offset between the LUMOs of the donor $(-3.29 \mathrm{eV})$ and the acceptors $(-3.74 \mathrm{eV}$ and $-3.68 \mathrm{eV})$, and suggests that the $V_{\mathrm{OC}}$ could be significantly improved by using a donor with a lower lying LUMO level. Secondly, we note that both blends show similarly high $\Delta V_{\mathrm{OC}, \mathrm{nrad}}$ of $0.36 \mathrm{~V}$ and $0.38 \mathrm{~V}$ for PFBDB-T:TTE-PDI4 and PFBDB-T:FTTE-PDI4, respectively, with the higher value for the FTTE-PDI4 blend meaning that the $V_{\mathrm{OC}}$ is not larger than that of the TTE-PDI4 blend despite the larger optical gap. An earlier study identified several molecular factors that could reduce the nonradiative voltage loss in solar cells, ${ }^{54}$ among which increased molecular rigidity, expressed as reduced reorganization energy, is one. Interestingly, whilst the higher molecular rigidity leading to the smaller reorganization energy of FTTE-PDI4 appears to facilitate charge transport in the blend (manifested through the higher FF), nonradiative recombination does not appear to be suppressed. It is therefore likely that the voltage losses are influenced also by microstructural properties of the blend films and not only by molecular parameters.

To confirm this hypothesis, atomic-force-microscopy (AFM) images were measured for both blends, as shown in Fig. 4. Both films display nanoscale phase separation with fine and small domain size, suggesting their twisted molecular geometry lead to the formation of favorable morphology for OPV devices. The root-mean-square (RMS) roughness are $3.0 \mathrm{~nm}$ and $7.6 \mathrm{~nm}$ for the PFBDB-T:TTE-PDI4 and PFBDB-T:FTTBPDI4 blends, respectively. As expected, the PFBDB-T:TTE-PDI4 blend shows strong intermixing and a smoother surface morphology, with fiber-like aggregates observed in the film, whilst the PFBDB-T:FTTEPDI4 blend shows more aggregated domains of roughly $25-30 \mathrm{~nm}$ in length, possibly due to the larger excess of acceptor in the optimized blend compared to in PFBDB-T:TTE-PDI4. This is likely to lead to a larger degree of disorder in the blend, which is consistent with the slightly larger $\Delta V_{\text {OC,nrad }}$ observed in this blend, although further measurements would be required to confirm this.

The molecular packing behaviour of compounds TTE-PDI4, FTTE-PDI4, PFBDB-T and their blend films were also investigated by X-ray diffraction on drop-cast films. As shown in Fig. S12 (ESI $\dagger$ ), a broad $\pi-\pi$ stacking peak was observed for 
Table 2 Photovoltaic parameters of the PFBDB-T:TTE-PDI4 and PFBDB-T:FTTE-PDI4 devices

\begin{tabular}{llllllll}
\hline Acceptor & $V_{\mathrm{OC}}(\mathrm{V})$ & $J_{\mathrm{SC}}\left(\mathrm{mA} \mathrm{cm}^{-2}\right)$ & $\mathrm{FF}(\%)$ & $\mathrm{PCE}^{a}(\%)$ & Calc. $J_{\mathrm{SC}}\left(\mathrm{mA} \mathrm{cm}^{-2}\right)$ & $V_{\mathrm{OC}, \text { rad }}(\mathrm{V})$ & $\Delta V_{\mathrm{OC}, \text { nrad }}(\mathrm{V})$ \\
\hline TTE-PDI4 & $0.99(0.99)$ & $7.5(7.3)$ & $51(50)$ & $3.8(3.6)$ & 8.2 & 1.35 \\
FTTE-PDI4 & $1.0(1.0)$ & $10.9(10.9)$ & $60(59)$ & $6.6(6.4)$ & 11.9 & 1.38 & 0.36 \\
\end{tabular}

${ }^{a}$ The values in parentheses are the average PCEs. The average values are collected from $>12$ independent devices.

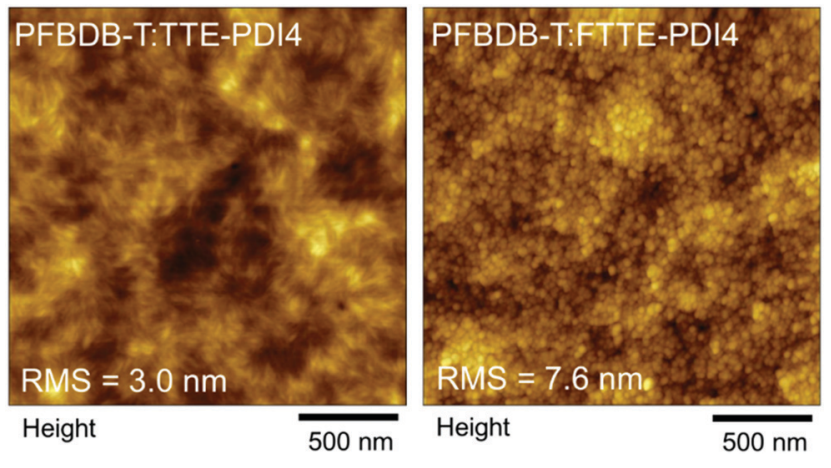

Fig. 4 AFM height images $(2 \mu \mathrm{m} \times 2 \mu \mathrm{m})$ of the PFBDB-T:TTE-PDI4 (left) and PFBDB-T:FTTBPDI4 (right) blend films. The topographical height difference $(\Delta z)$ are $21 \mathrm{~nm}$ and $51 \mathrm{~nm}$, respectively.

neat PFBDB-T film, with a maxima at $2 \theta=24.26^{\circ}$. After annealing at $160{ }^{\circ} \mathrm{C}$ for $10 \mathrm{~min}$, the film not only exhibited a $\pi-\pi$ stacking at a similar position, but also weak diffraction peaks at $2 \theta=12.72^{\circ}, 14.14^{\circ}, 18.60^{\circ}$ and $21.68^{\circ}$, suggestive of enhanced order. The enhanced order after thermal annealing is in agreement with our previous GIWAXS results on thin-films. ${ }^{6}$ TTEPDI4 and FTTE-PDI4 exhibited no diffraction peaks, indicating their low crystallinity in thin films, which was also consistent with DSC data. The optimal blend films of PFBDB-T:TTE-PDI4 and PFBDB-T:FTTE-PDI4 also showed no diffraction peaks from their drop cast blends after annealing at $140{ }^{\circ} \mathrm{C}$ for $10 \mathrm{~min}$, suggesting blending may inhibit polymer crystallisation somewhat, with the caveat that any crystallites present may not be readily observed with the lab based diffractometer used in these measurements.

\section{Conclusions}

In conclusion, two PDI tetramers with non-fused and fused structures, TTE-PDI4 and FTTE-PDI4, were designed and synthesized. DFT calculations reveal that the fused FTTE-PDI4 displays reduced intramolecular twisting compared to nonfused TTE-PDI4 with a lower internal reorganization energy. FTTE-PDI4 exhibits a smaller band gap than TTE-PDI4, with a much broader and stronger absorption in the region of 300-600 nm, together with an up-shifted LUMO level. TD-DFT calculations confirm that the observed strong absorption features at low wavelengths, also reported in other fused-PDI molecules in the literature, are due to multiple bright electronic states coupled to a strong vibrational band at $1600 \mathrm{~cm}^{-1}$. Consequently, the PFBDB-T:FTTE-PDI4 devices achieved a highest PCE of $6.6 \%$ with a higher current density of $10.9 \mathrm{~mA} \mathrm{~cm}^{-2}$ and $\mathrm{FF}$ of $60 \%$. This work demonstrates that ring fusion is an effective strategy to broaden the absorption spectra of PDI-type OPVs by induced bright low-lying bright electronic states, as well as finely tune the molecular shape, both of which work to boost device efficiency.

\section{Conflicts of interest}

There are no conflicts to declare.

\section{Acknowledgements}

We thank the China Scholarship Council (CSC) via the CSC Imperial Scholarship, Engineering and Physical Sciences Research Council (EPSRC) (Grant EP/L016702/1) and the Royal Society and the Wolfson Foundation (Royal Society Wolfson Fellowship) for funding. F. E. thanks the Engineering and Physical Sciences Research Council (EPSRC) for support via the Post-Doctoral Prize Fellowship, and J. N. and F. E. are grateful for funding from the European Research Council (ERC) under the European Union's Horizon 2020 research and innovation program (grant agreement no. 742708). The authors also thank Filip Aniés, Zhongyao Jiang and Richard Sweeney for valuable help.

\section{Notes and references}

1 C. Yan, S. Barlow, Z. Wang, H. Yan, A. K. Y. Jen, S. R. Marder and X. Zhan, Nat. Rev. Mater., 2018, 3, 18003.

2 J. Zhang, H. S. Tan, X. Guo, A. Facchetti and H. Yan, Nat. Energy, 2018, 3, 720-731.

3 J. Hou, O. Inganas, R. H. Friend and F. Gao, Nat. Mater., 2018, 17, 119-128.

4 A. Wadsworth, M. Moser, A. Marks, M. S. Little, N. Gasparini, C. J. Brabec, D. Baran and I. McCulloch, Chem. Soc. Rev., 2018, 48, 1596-1625.

5 P. Cheng, G. Li, X. Zhan and Y. Yang, Nat. Photonics, 2018, 12, 131-142.

6 Z. Fei, F. D. Eisner, X. Jiao, M. Azzouzi, J. A. Röhr, Y. Han, M. Shahid, A. S. R. Chesman, C. D. Easton, C. R. McNeill, T. D. Anthopoulos, J. Nelson and M. Heeney, Adv. Mater., 2018, 30, 1705209.

7 L. Lu, T. Zheng, Q. Wu, A. M. Schneider, D. Zhao and L. Yu, Chem. Rev., 2015, 115, 12666-12731.

8 Z. Liu, Y. Wu, Q. Zhang and X. Gao, J. Mater. Chem. A, 2016, 4, 17604-17622. 
9 Y. Lin, F. Zhao, Q. He, L. Huo, Y. Wu, T. C. Parker, W. Ma, Y. Sun, C. Wang, D. Zhu, A. J. Heeger, S. R. Marder and X. Zhan, J. Am. Chem. Soc., 2016, 138, 4955-4961.

10 X. Guo, A. Facchetti and T. J. Marks, Chem. Rev., 2014, 114, 8943-9021.

11 J. Wang and X. Zhan, Trends Chem., 2019, 1, 869-881.

12 C. W. Tang, Appl. Phys. Lett., 1986, 48, 183-185.

13 W. Zhu, J. M. Alzola, T. J. Aldrich, K. L. Kohlstedt, D. Zheng, P. E. Hartnett, N. D. Eastham, W. Huang, G. Wang, R. M. Young, G. C. Schatz, M. R. Wasielewski, A. Facchetti, F. S. Melkonyan and T. J. Marks, ACS Energy Lett., 2019, 4, 2695-2702.

14 Q. Wu, D. Zhao, A. M. Schneider, W. Chen and L. Yu, J. Am. Chem. Soc., 2016, 138, 7248-7251.

15 H. Yu, L. Arunagiri, L. Zhang, J. Huang, W. Ma, J. Zhang and H. Yan, J. Mater. Chem. A, 2020, 8, 6501-6509.

16 C. Li and H. Wonneberger, Adv. Mater., 2012, 24, 613-636.

17 J. E. Anthony, A. Facchetti, M. Heeney, S. R. Marder and X. Zhan, Adv. Mater., 2010, 22, 3876-3892.

18 S. Li, W. Liu, C.-Z. Li, M. Shi and H. Chen, Small, 2017, 13, 1701120.

19 Q. He, T. Li, C. Yan, Y. Liu, J. Wang, M. Wang, Y. Lin and X. Zhan, Dyes Pigm., 2016, 128, 226-234.

20 C. Zhan and J. Yao, Chem. Mater., 2016, 28, 1948-1964.

21 C. B. Nielsen, S. Holliday, H.-Y. Chen, S. J. Cryer and I. McCulloch, Acc. Chem. Res., 2015, 48, 2803-2812.

22 Y. Zhong, M. T. Trinh, R. Chen, W. Wang, P. P. Khlyabich, B. Kumar, Q. Xu, C.-Y. Nam, M. Y. Sfeir, C. Black, M. L. Steigerwald, Y.-L. Loo, S. Xiao, F. Ng, X. Y. Zhu and C. Nuckolls, J. Am. Chem. Soc., 2014, 136, 15215-15221.

23 D. Meng, D. Sun, C. Zhong, T. Liu, B. Fan, L. Huo, Y. Li, W. Jiang, H. Choi, T. Kim, J. Y. Kim, Y. Sun, Z. Wang and A. J. Heeger, J. Am. Chem. Soc., 2016, 138, 375-380.

24 Q. Yan, Y. Zhou, Y.-Q. Zheng, J. Pei and D. Zhao, Chem. Sci., 2013, 4, 4389-4394.

25 Y. Liu, C. Mu, K. Jiang, J. Zhao, Y. Li, L. Zhang, Z. Li, J. Y. L. Lai, H. Hu, T. Ma, R. Hu, D. Yu, X. Huang, B. Z. Tang and H. Yan, Adv. Mater., 2015, 27, 1015-1020.

26 S. Li, W. Liu, C.-Z. Li, F. Liu, Y. Zhang, M. Shi, H. Chen and T. P. Russell, J. Mater. Chem. A, 2016, 4, 10659-10665.

27 Y. Duan, X. Xu, H. Yan, W. Wu, Z. Li and Q. Peng, Adv. Mater., 2017, 29, 1605115.

28 H. Lin, S. Chen, H. Hu, L. Zhang, T. Ma, J. Y. L. Lai, Z. Li, A. Qin, X. Huang, B. Tang and H. Yan, Adv. Mater., 2016, 28, 8546-8551.

29 A. Kuzmich, D. Padula, H. Ma and A. Troisi, Energy Environ. Sci., 2017, 10, 395-401.

30 H. Zhong, C.-H. Wu, C.-Z. Li, J. Carpenter, C.-C. Chueh, J.-Y. Chen, H. Ade and A. K. Y. Jen, Adv. Mater., 2016, 28, 951-958.

31 H. Qian, Z. Wang, W. Yue and D. Zhu, J. Am. Chem. Soc., 2007, 129, 10664-10665.

32 Y. Zhong, M. T. Trinh, R. Chen, G. E. Purdum, P. P. Khlyabich, M. Sezen, S. Oh, H. Zhu, B. Fowler, B. Zhang, W. Wang, C.-Y. Nam, M. Y. Sfeir, C. T. Black, M. L. Steigerwald, Y.-L. Loo, F. Ng, X. Y. Zhu and C. Nuckolls, Nat. Commun., 2015, 6, 8242.
33 D. Meng, H. Fu, C. Xiao, X. Meng, T. Winands, W. Ma, W. Wei, B. Fan, L. Huo, N. L. Doltsinis, Y. Li, Y. Sun and Z. Wang, J. Am. Chem. Soc., 2016, 138, 10184-10190.

34 Q. Wu, D. Zhao, J. Yang, V. Sharapov, Z. Cai, L. Li, N. Zhang, A. Neshchadin, W. Chen and L. Yu, Chem. Mater., 2017, 29, 1127-1133.

35 J. Zhang, Y. Li, J. Huang, H. Hu, G. Zhang, T. Ma, P. C. Y. Chow, H. Ade, D. Pan and H. Yan, J. Am. Chem. Soc., 2017, 139, 16092-16095.

36 G. Zhang, J. Feng, X. Xu, W. Ma, Y. Li and Q. Peng, Adv. Funct. Mater., 2019, 29, 1906587.

37 Y. Firdaus, V. M. Le Corre, J. I. Khan, Z. Kan, F. Laquai, P. M. Beaujuge and T. D. Anthopoulos, Adv. Sci., 2019, 6, 1802028.

38 N. C. Davy, M. Sezen-Edmonds, J. Gao, X. Lin, A. Liu, N. Yao, A. Kahn and Y.-L. Loo, Nat. Energy, 2017, 2, 17104.

39 L. Viglianti, N. L. C. Leung, N. Xie, X. Gu, H. H. Y. Sung, Q. Miao, I. D. Williams, E. Licandro and B. Z. Tang, Chem. Sci., 2017, 8, 2629-2639.

40 J.-J. Liu, J. Yang, J.-L. Wang, Z.-F. Chang, B. Li, W.-T. Song, Z. Zhao, X. Lou, J. Dai and F. Xia, Mater. Chem. Front., 2018, 2, 1126-1136.

41 A. Yamamoto, Y. Matsui, T. Asada, M. Kumeda, K. Takagi, Y. Suenaga, K. Nagae, E. Ohta, H. Sato, S. Koseki, H. Naito and H. Ikeda, J. Org. Chem., 2016, 81, 3168-3176.

42 Y. Zhong, B. Kumar, S. Oh, M. T. Trinh, Y. Wu, K. Elbert, P. Li, X. Zhu, S. Xiao, F. Ng, M. L. Steigerwald and C. Nuckolls, J. Am. Chem. Soc., 2014, 136, 8122-8130.

43 J. Zhang, F. Bai, Y. Li, H. Hu, B. Liu, X. Zou, H. Yu, J. Huang, D. Pan, H. Ade and H. Yan, J. Mater. Chem. A, 2019, 7, 8136-8143.

44 J. Seibt, P. Marquetand, V. Engel, Z. Chen, V. Dehm and F. Würthner, Chem. Phys., 2006, 328, 354-362.

45 N. J. Schuster, D. W. Paley, S. Jockusch, F. Ng, M. L. Steigerwald and C. Nuckolls, Angew. Chem., Int. Ed., 2016, 55, 13519-13523.

46 A. Oleson, T. Zhu, I. S. Dunn, D. Bialas, Y. Bai, W. Zhang, M. Dai, D. R. Reichman, R. Tempelaar, L. Huang and F. C. Spano, J. Phys. Chem. C, 2019, 123, 20567-20578.

47 L. Ye, S. Zhang, W. Zhao, H. Yao and J. Hou, Chem. Mater., 2014, 26, 3603-3605.

48 Y. Liu, J. Zhao, Z. Li, C. Mu, W. Ma, H. Hu, K. Jiang, H. Lin, H. Ade and H. Yan, Nat. Commun., 2014, 5, 5293.

49 D. Qian, L. Ye, M. Zhang, Y. Liang, L. Li, Y. Huang, X. Guo, S. Zhang, Z. A. Tan and J. Hou, Macromolecules, 2012, 45, 9611-9617.

50 F. D. Eisner, M. Azzouzi, Z. Fei, X. Hou, T. D. Anthopoulos, T. J. S. Dennis, M. Heeney and J. Nelson, J. Am. Chem. Soc., 2019, 141, 6362-6374.

51 U. Rau, B. Blank, T. C. M. Müller and T. Kirchartz, Phys. Rev. Appl., 2017, 7, 044016.

52 J. Yao, T. Kirchartz, M. S. Vezie, M. A. Faist, W. Gong, Z. He, H. Wu, J. Troughton, T. Watson, D. Bryant and J. Nelson, Phys. Rev. Appl., 2015, 4, 014020.

53 U. Rau, Phys. Rev. B: Condens. Matter Mater. Phys., 2007, 76, 085303.

54 M. Azzouzi, J. Yan, T. Kirchartz, K. Liu, J. Wang, H. Wu and J. Nelson, Phys. Rev. X, 2018, 8, 031055. 